\title{
Mobile Robot in Coal Mine Disaster Surveillance
}

\author{
Mr. Sabarish Chakkath ${ }^{1}$, S.Hariharansiddharath ${ }^{2}$, B.Hemalatha ${ }^{3}$ \\ ${ }^{1}$ Student, ${ }^{2}$ Student, ${ }^{3}$ Assistant professor Velammal Engineering.College Chennai, Tamilnadu, India
}

\begin{abstract}
Coal mine disaster is a common occurrence in mines after which rescue operations are immediately needed which is a risky one. The disaster results in increased level of harmful gases like CO and $\mathrm{CO} 2$ resulting in decreased $\mathrm{O} 2$ level and high temperature. Moreover the explosion might recur, making direct human intervention impossible inside the tunnel for rescue operation. To prevent human and material loss after mine explosion, a Robot is deployed inside the tunnel, which can travel inside the tunnel to sense gas contents, temperature and transmit the signals to the control room. The robot also has a wireless camera which will transmit live video signal to monitor the status inside the tunnel and to control the movement of the robot. The robot is a conveyor belt type robot enabling it to climb up the obstacles and move across debris. The ZigBee technology is used along with RF transceivers to carry out cheap and efficient wireless communication. With the help of this mobile robot, the loss due to coal mine disaster can be reduced and efficient rescue operation can be carried out.
\end{abstract}

Key words - Coal mine, Disaster surveillance, Robot, Sensor

\section{INTRODUCTION}

Coal mine is a special type of mine which is dangerous in nature. Such mines will usually be an underground system which has less number of pit heads. In case of any accident as given in Table 1, it will be difficult for people to escape out of the mine. The dangerous factors that an accident will bring along are collapse of tunnel, gas explosion, undesired level of $\mathrm{O} 2, \mathrm{CO}$ and $\mathrm{CO} 2$, high temperature, fire break out, etc. All these factors can cause human and property loss. These fatal factors menace people. After a Coal mine disaster, the situation inside the mine is not known. Entering into a mine without knowing the exact situation is dangerous as the chances of a second explosion is high. Many rescuers are killed by this second explosion. Detection of the situation inside the tunnel is of high importance.

The problem in the tunnel is that it is narrow and rugged. The tunnel's middle section has a rail track and to its one side it has belt transmission and to the other side it has a narrow path of coal. Due to this the transmission along the tunnel is a difficult thing. This is the condition of the normal tunnel. When an explosion occurs, the situation inside the tunnel worsens and the conveyance inside the tunnel becomes really difficult. To overcome such hazardous situation, a robot can be deployed for disaster surveillance.

Table 1.Statistics Analysis of Death Accident in Coal Mines[1].

\begin{tabular}{|l|l|l|}
\hline Accident type & Occurrence & Death count \\
\hline Gas accidents & 3318 & 492 \\
\hline Cavings & 1332 & 907 \\
\hline Flood Accidents & 1111 & 166 \\
\hline Transportation & 261 & 230 \\
\hline Dust & 234 & 4 \\
\hline Gun fire & 58 & 39 \\
\hline Collapse & 57 & 14 \\
\hline Electricity & 40 & 38 \\
\hline
\end{tabular}

The accidents due to gas leakage, dust, collapse and transportation can be overcome convincingly by using the mobile robot. The gas sensors will indicate the presence of harmful gases and it will alert the workers to be better prepared to tackle the situation. This is the main objective of using the robot, to reduce human and material loss. 


\section{LITERATURE REVIEW}

The staggering data from [1] reveals that huge number of accidents occur in a coal mine during and after a disaster. The main reasons being, gas accidents, caving, flood, etc. This really asks for the development of a system that can help minimize the human and material loss that happens during rescue operations. Gas explosion is the most serious one in all mine disasters for after the gas explosion, the scene becomes extremely complex [2]. Thus gas sensors are mandatory in the robot to be deployed.

The idea of a Mobile Robot to be able to aid the rescue team entering into a coal mine[3] got picked up with the tremendous uplift in the field of technology. The Robot is used to reach the disaster zone and it is used for rescue and research operations. The robot can go into explosion environment and detect gas contents, temperature, etc. The data can be sent to controller in safe field. The robot is designed in such a way that it suits the mine tunnel. It can run in explosion environment, climb over ruins, check gas and if needed, slot can be provided wherein it can carry food and first aid kit to the workers trapped inside.

A biped robot was initially tipped to be used in the coal mines[4] which can move inside the tunnel and has an RS 232 cable for interfacing. The robot couldn't transverse a long distance it tripped on moving across the debris and the RS 232 cables couldn't be used for a long distance. Thus a different mode of legs had to be used and after research, the usage of a conveyor belt type robot was developed. The conveyor belt as seen on the military tanks would maneuver over debris and rough terrains.

Now, the focus was onto the means of communication to transfer data and commands to and fro from computer and robot. In the earlier days, the robot had RS 232 cables to provide communication between the robot and the CPU[4]. The RS-232 as the interface for communication and control to allow the robot to receive demands from the user and track objects autonomously. Though the RS 232 gave creditable communication, it had several short comings. Due to its limited size, the robot was not able to move deep into the mine. And there was the danger of the cables getting twinned or cut inside the tunnel resulting in loss of data or at times, it can spark a fire inside the tunnel. A better way of communication had to be used.

The advancement in technology saw the usage of Blue Tooth for wireless communication [5]. The mobile robot has the capability to move around autonomously using complicated and powerful algorithm. The algorithms are stored in a PC that acts as a master cum server. All sensor readings from the mobile robot will be transmitted to the master and processed. Then, command or instruction for further action is transmitted from the server to the mobile robot in a bidirectional full duplex communication mode. Hence, the main "brain" is in the server instead of the mobile robot. This again had the shortcoming of limited coverage area. The Blue Tooth would function only to a specified area and the robot couldn't be controlled beyond that.

This lead to the usage of much wide wireless communications technologies like RF transceiver and ZigBee[6]. The ZigBee had much better range than Blue Tooth and hence it could transmit commands and receive data from long distance from the tunnel.

Once the communication and movement and sensing of robot was finalized, the focus shifted on how to make it more reliable. The idea to use a camera on the robot helps in getting live feed of what is happening inside the tunnel and it will help the rescue team to follow the less dangerous path. i.e, it helps in mapping of the mine. Robot wireless sensor networks are an increasingly capable platform and show great potential in environments from future battlefields to disaster discovery. Robot wireless sensor networks can enhance multimedia surveillance and provide the foundation for strategies based on multi-modal sensor integration [7].

The IEEE paper authored by Shan Cai, Zhao-long, Xu Jie, Yang Xiao-zhi Liu et al [8] concerns a robot system fit for detecting mine disaster information on the basis of the working principle of detecting robot system. It introduces in details the structure of the system; the technologies of passing through obstacles, obstacles avoidance by using sensors; the collection of information and wireless transmission modules, including gas sensors, temperature sensors, network cameras and wireless transmission modules.

\section{ARCHITECTURE}

The architrctural diagram shown in Fig.1 comprises of Microcontroller, sensor unit, camera unit, motor driver unit, mechanical driver unit and a communication medium $(2.4 \mathrm{GHz})$. 


\section{ROBOT UNIT:}
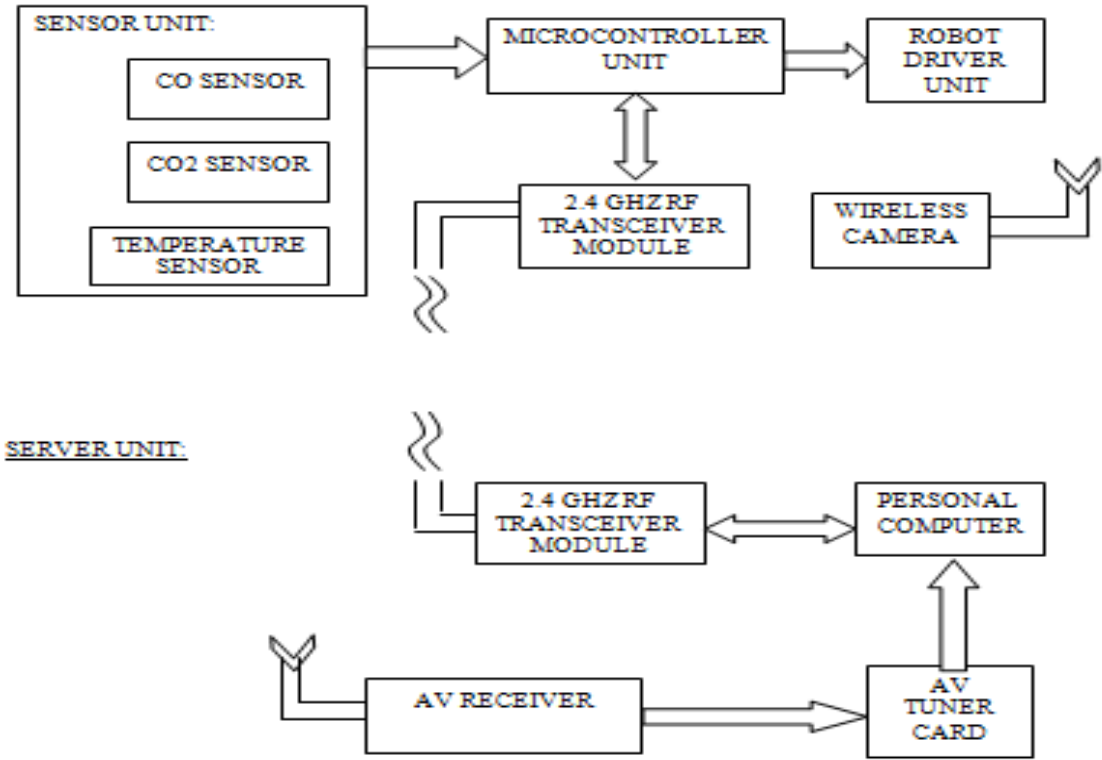

Figure 1.Architecture of mobile robot

The sensor unit must be directly interfaced to the controller. The monitoring device takes the current readings and sends them to the Microcontroller. The controller circuit is responsible for transmitting this information. Controllers are designed at hardware level. The $2.4 \mathrm{GHz}$ module is interfaced with the controller via the standard serial port. Readings are periodically reported to monitoring system in the form of radio packet service. These data's are updated by the PC/SERVER. The readings about the parameters of the mine will be thus updated and automatically displayed on the monitoring system. So that rescue team can view the readings continuously. And motion detection camera is used to visualize the critical situation of the mine.

\subsection{SENSOR UNIT}

A sensor (also called detector) is a converter that measures a physical quantity and converts it into a signal which can be read by an observer or by an instrument. Three sensors are used in the project. They are CO2, CO and Temperature Sensors for which MQ-6, MQ-7, and LM35 sensors are used respectively. The MQ6 and MQ-7 can detect gas concentrations anywhere from 200 to 10000 ppm. The LM 35 has a range of $0-110$ degree Celsius. The three sensors module is designed using Proteus ISIS and the soldering of the sensors into the PCB is done. The values read by the sensors are transmitted to a PIC 16F877A wherein it compares the measured value with that of the set points and if the measured value goes above the set points, the observer will be made aware of it by the window saying "CO level has exceeded". This helps in having good knowledge about the environment. And a wireless video camera is used to track the happenings inside the tunnel. The sensor circuit comprising of the three sensors is given in Fig. 2.

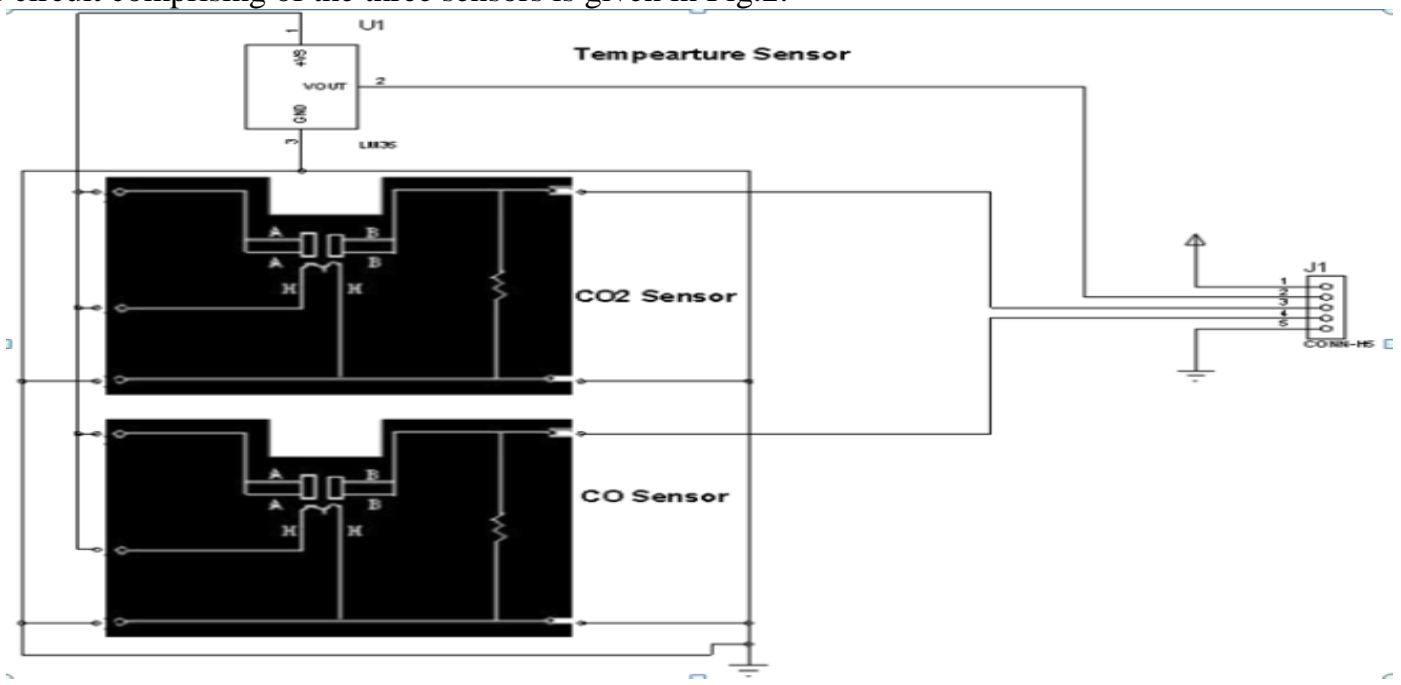

Figure 2.Sensor circuit 


\subsection{Microcontroller unit}

The microcontroller that is been used is the PIC 16F877A controller. PICs are popular with both industrial developers and hobbyists alike due to their low cost, wide availability, large user base, extensive collection of application notes, availability of low cost or free development tools, and serial programming (and re-programming with flash memory) capability. The microcontroller is used to collect the parameter value from the sensor unit and compare it with the set point (safer level of gases) and transfer the corresponding data to the CPU. It also receives commands from the CPU and transfers it to the robot unit for its movement. The microcontroller is the core of the surveillance robot.

\subsection{Robot driver unit}

The robot driver unit is primarily concerned about the movement of the robot in $\mathrm{x}$-axis and $\mathrm{y}$-axis. The robot is of conveyor belt type as it helps to maneuver over debris and rugged terrain. The wheels are run by two DC motors of 200 rpm. When both the wheels are given with positive pulse, the robot moves in forward direction, when the supply is reversed, it goes in backward direction and similarly by varying the negative and positive, left and right turn can be achieved. To choose the supply given to each motor, L293D IC is used. The robot movement circuit is given in fig. 3 which will drive the robot to move in forward, reverse and turn left and right.

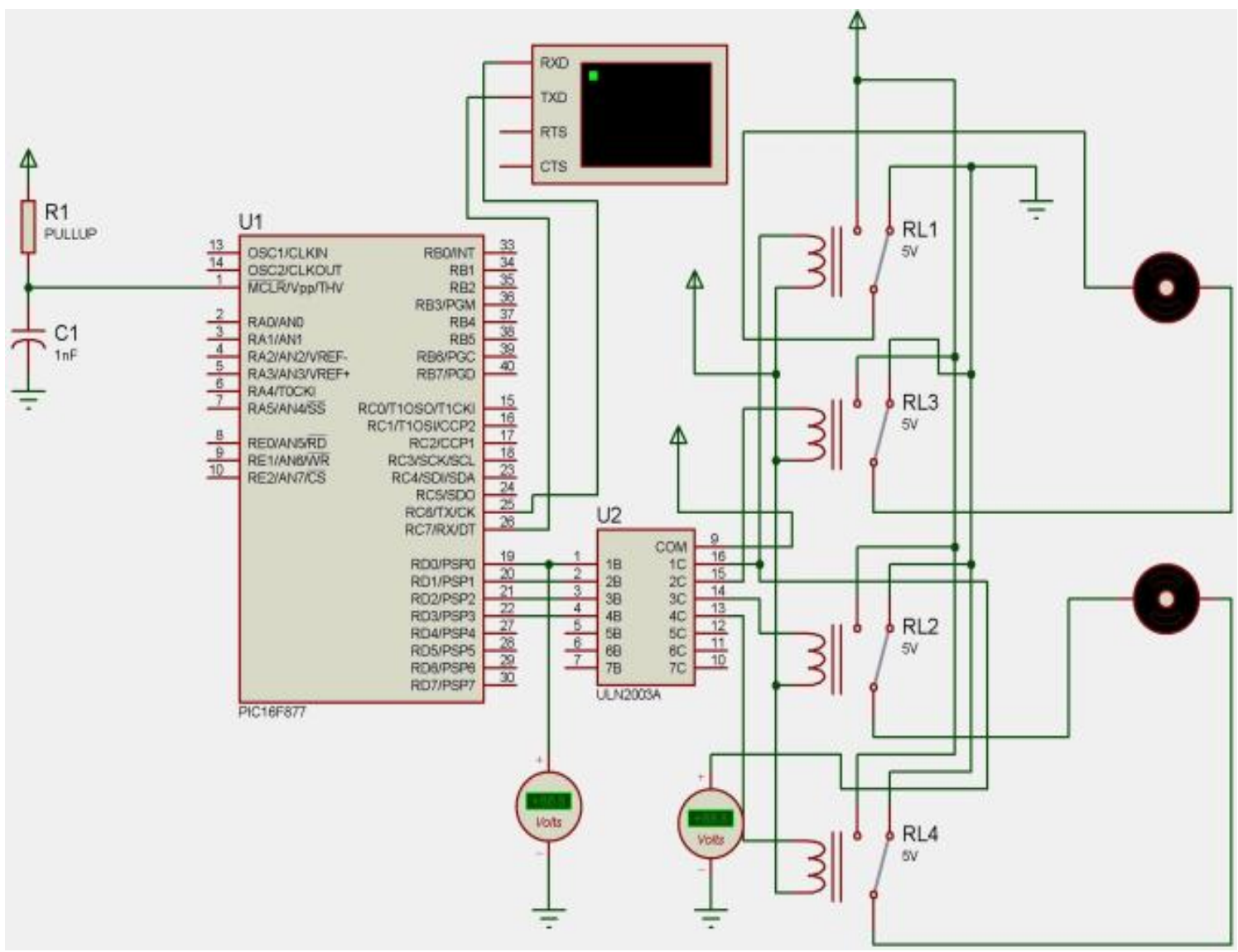

Figure 3.Robot movement unit

\subsection{Transciever unit}

The transceiver unit is used to both transmit and receive data. The 2.4GHZ RF transceiver is used as it offers free bandwidth. To improve the security, other secure frequencies can be used. One transceiver is mounted on the robot unit, which gets the data from the microcontroller and transmits to the other transceiver placed in the control room. Similarly, the transceiver placed in the control room will transmit commands to the transceiver mounted on the robot for the control of robot movement.

\subsection{Camera module}

The camera module consists of a wireless camera and an AV receiver and an AV tuner card. The wireless camera is mounted on the robot and the video signal is transmitted to the AV receiver and it is tuned to get the original signal. The video is then transmitted to the CPU by interfacing an AV tuner card. The camera 
module will transmit the video coverage of the paths and thus helping in easier mapping of the path to be taken by the rescue team. For real time applications, wireless camera of high range is to be used to get good clarity and good coverage. The camera employed will also help the robot from getting stuck in a pit as the obstacles lying in path is foreseen and required action can be taken, thus improving the life of robot inside the tunnel.

\section{RESULTS AND DISCUSSION}

The mobile robot for mine disaster surveillance is designed and the robot looks as shown in fig.4 with its camera and other components attached to it. A $12 \mathrm{~V} / 5 \mathrm{~A}$ rechargeable battery is used to run the robot. The robot was test run and it moved well on debris and over rough terrains and the video was transmitted with much clarity.

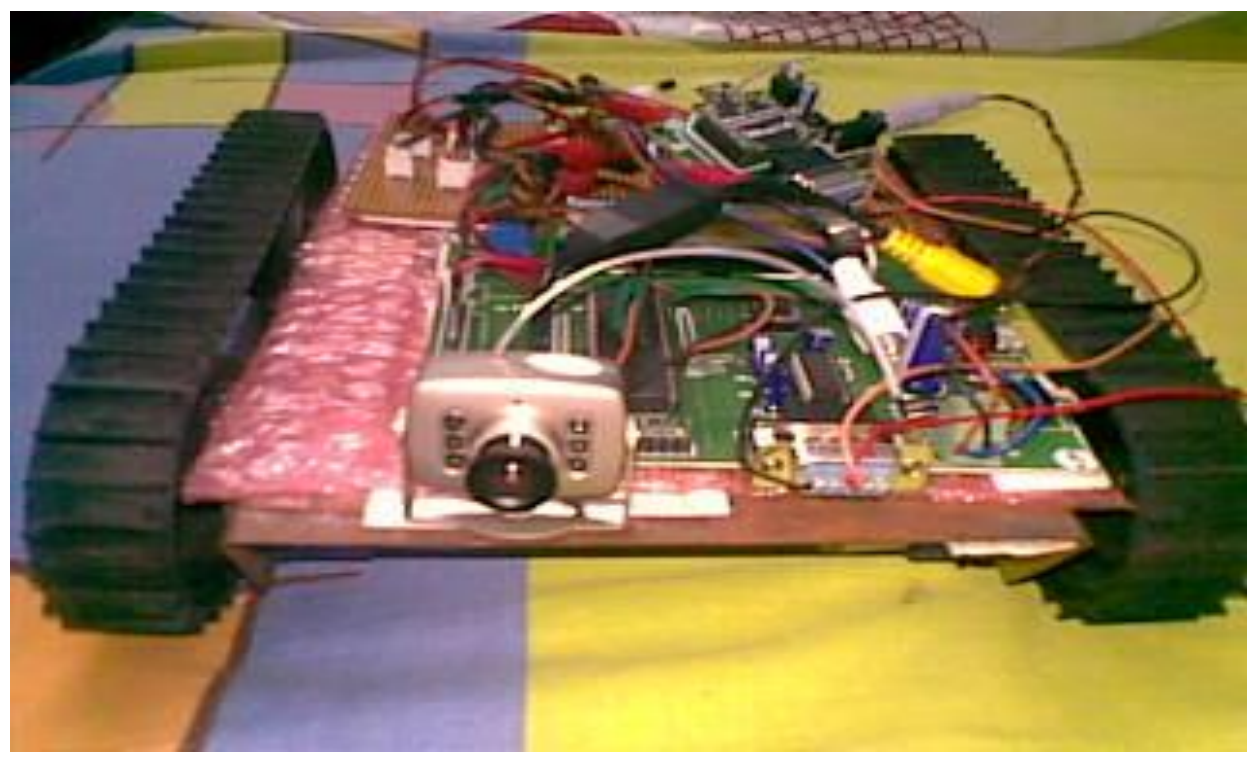

Figure 4. Prototype of mobile robot

The sensors are tested for their performance and to conduct the test, a wick or any other source of smoke is brought near to the MQ 7 and MQ 6 sensor. This prototype robot has its safe values of CO and CO2 set at $10 \mathrm{ppm}$ and $60 \mathrm{ppm}$. The set point for temperature is 40 degree Celsius. Once the measured value goes beyond these set points, a window will pop out in the CPU showing the parameters' level has exceeded as shown in Fig.5.

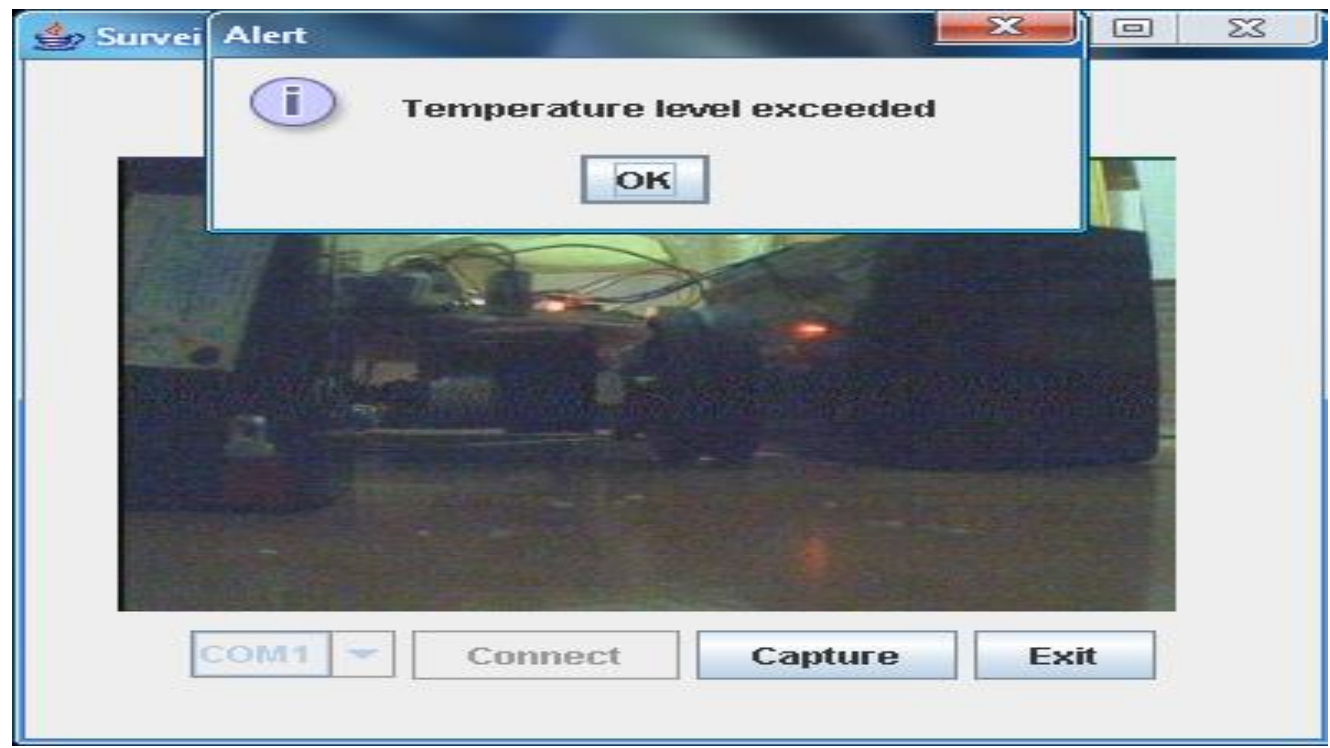

Figure 5. User end screen 


\section{CONCLUSION}

The project is aimed at providing human safety for the rescue team in hazardous environments such as coal mines . This is a prototype which can be implemented in real time by using components with better range and efficiency. This robot enters into hazardous environments and provides data like the content of various gases after the explosion has occurred and also the temperature based on which the rescue team will be sent with necessary precautionary measures in order to make sure that the rescue team does not come to any harm. The conveyor belt enables the robot to run in rugged environment overcoming the disadvantage of armed robots as they might trip and fall in such conditions.

In future this can be developed by the use of higher transmission range transceivers so that it can travel for a greater distance and can be used in different environments based on the transmission range. Development can also be made in the number of sensors incorporated in the robot. Various other sensors like LPG sensor, O2 sensor can be added and thus helping to get a much improved image of the environment inside. Implantation of an arm on the robot can help the robot pick up samples or remove small debris from path or open or close any doors if needed inside the mine.

\section{REFERENCE}

[1] Liu Xiaoli, Guo Liwen, and Zhang Zhiye, Statistics Analasis of death Accident in Coal Mines from January 2005 to June 2009; IEEE 2010

[2] Zhou, Xin-quan, and Chen Guo-xin. The probability analysis of occurrence causes of extraordinarily serious gas explosion accidences and its revelation. Journal of China Coal Society, 2008, 33 (1): 42-46.

[3] GAO junyao, GAO xueshan, ZHU wei, ZHUjianguo, WEI boyu. 'Coal Mine Detect and Rescue Robot Design and Research'.IEEE 2008

[4] Jong C. Wang, Yan Ting Lin, Huei Teng Jheng, Jyun Sian Wu and Ruei Jhe Li, Object Tracking for Autonomous Biped Robot. IEEE 2010

[5] H. Choo', Shamsudin H.M.Amin', N. Fisal', C. F. Yeong', and J. Abu Bakar, Using blue tooth transceivers in mobile robot. 2002 Student Conference on Research and Development Proceedings, Shah Alam, Malaysia.

[6] M. Thamrin N., Rosman R, and Sarmawi D. S,Design and Analysis of Wireless Controller Panel using RF Module's for Robotic Wheelchair. IEEE 2011.

[7] Heng Huanga, Fillia Makedona, Dan Popab, and Harry Stephanoub, A feature extraction method for multimedia data analysis in robot wireless sensor networks. IEEE 2007

[8] Shan Cai, Zhao-long, Xu Jie Yang, and Xiao-zhi Liu, Detecting Robot System for Mine Disasters. ICEEE 2010 